\title{
Evaluation of the Learning Curve for Conformal Sphincter Preservation Operation in The Treatment of Ultra-Low Rectal Cancer
}

Hai-bo ding ( $2812097576 @ q q . c o m$ )

Changhai Hospital https://orcid.org/0000-0002-7259-6654

\section{Lin-hui Wang}

Zhejiang University College of Education

\section{Ge Sun}

University Medical Centre Groningen: Universitair Medisch Centrum Groningen

\section{Guan-yu Yu}

Changhai Hospital

\section{Xian-hua Gao}

Changhai Hospital

\section{Kuo Zheng}

Changhai Hospital

\section{Hai-feng Gong}

Changhai Hospital

Jin-ke Sui

Changhai Hospital

Xiao-ming Zhu

Changhai Hospital

\section{Wei Zhang}

Changhai Hospital https://orcid.org/0000-0003-3094-9711

\section{Research Article}

Keywords: Learning curve, Ultra-low rectal cancer, Conformal sphincter preservation operation, Operation time

Posted Date: December 10th, 2021

DOI: https://doi.org/10.21203/rs.3.rs-1119399/v1

License: (c) (1) This work is licensed under a Creative Commons Attribution 4.0 International License.

Read Full License 


\section{Abstract}

Background: To investigate the learning curve of conformal sphincter preservation operation (CSPO) in the treatment of ultra-low rectal cancer and to further expore the influencing factors of operation time.

Methods: From August 2011 to April 2020, 108 consecutive patients with ultra-low rectal cancer underwent CSPO by the same surgeon in the department of colorectal surgery of Changhai Hospital. The moving average and cumulative sum control chart (CUSUM) curve were used to analyze the learning curve. The preoperative clinical baseline data, postoperative pathological data, postoperative complications and survival data were compared before and after the completion of learning curve. The influencing factors of CSPO operation time were analyzed by univariate and multivariate analysis.

Results: According to the results of moving average and CUSUM method, CSPO learning curve was divided into learning period (1-45 cases) and learning completion period (46-108 cases). There was no significant difference in preoperative clinical baseline data, postoperative pathological data, postoperative complications and survival data between the two stages. Compared with the learning period, the operation time $(P<0.05)$, blood loss $(P<0.05)$, postoperative flatus and defecation time $(P<$ $0.05)$, liquid diet time $(P<0.05)$ and postoperative hospital stay $(P<0.05)$ in the learning completion period were significantly reduced, and the difference was statistically significant. Univariate and multivariate analysis showed that distance of tumor from anal verge ( $\geq 4 \mathrm{~cm}$ vs. $<4 \mathrm{~cm}, P=0.039)$ and $\mathrm{T}$ stage (T3 vs. T1-2, $P=0.022$ ) were independent risk factors for prolonging the operation time of CSPO.

Conclusions: For surgeons with laparoscopic surgery experience, about 45 cases of CSPO are needed to cross the learning curve. At the initial stage of CSPO, beginners are recommended to select patients with ultra-low rectal cancer whose distance of tumor from anal verge is less than $4 \mathrm{~cm}$ and tumor stage is less than T3 for practice, which can enable beginners to reduce the operation time, accumulate experience, build self-confidence and shorten the learning curve on the premise of safety.

\section{Background}

Miles operation [1] is considered as the standard treatment for ultra-low rectal cancer with the lower edge of the tumor less than $2 \mathrm{~cm}$ from the dentate line. However, the stomal complications [2] and long-term nursing of permanent colostomy have seriously reduced the quality of life [3], role and social function [4] of patients after Miles. Hence, many patients with ultra-low rectal cancer refuse colostomy before operation [5]. In recent years, preoperative neoadjuvant chemoradiotherapy (nCRT) [6-7] and laparoscopic technology have developed rapidly. People also have a deeper understanding of the safety of rectal cancer with a distance of less than $2 \mathrm{~cm}$ from the distal resection margin [8]. The maturity of technique and anatomic basis of anal-preserving operation not only improves its oncological prognosis [9] and functional prognosis [10], but also promotes the expansion of its related research and application.

Considering the anatomic characteristics of the anal canal, Department of Colorectal Surgery, Shanghai Changhai Hospital proposed the CSPO [11-13] for ultra-low rectal cancer. As a new anal-preserving 
operation, it combined with total mesorectal excision [14-15] (TME), coloanal anastomosis [16], eversion pull-through resection [17], anal cannal dissection, local tumor resection [18], natural orifice translumenal endoscopic surgery [19] (NOTES) and so forth. Since August 2011, our group has successfully passed the CSPO learning curve and achieved satisfactory results. By April 2020, we have completed 123 cases of CSPO. The favorable oncology and functional prognosis of patients with CSPO has gradually been confirmed by relevant studies in our center and relevant literature has also been published [11-13].

As a new anal-preserving operation, CSPO has deep experience requirements for laparoscopic surgery, perianal disease surgery and manual coloanal anastomosis. To ensure the safety of CSPO, it is important to evaluate its learning curve and establish an appropriate training program. However, the learning curve of CSPO is not clear at present. In this study, we evaluated the learning curve by analyzing the clinical data of 108 consecutive patients who underwent laparoscopic CSPO by a same surgeon. By moving average, CUSUM, we systematically evaluated the learning curve of CSPO, and further analyzed the influencing factors of CSPO operation time.

\section{Methods}

\section{Patients}

From August 2011 to April 2020, 123 consecutive patients with ultra-low rectal cancer successively implemented CSPO by the same surgeon of colorectal surgery department of Shanghai Changhai Hospital, China. The surgeon had experience of performing more than 200 cases of routine laparoscopic surgery for colorectal cancer over 10 years. The operation time was used as the main index for the evaluation of learning curve. In addition to CSPO, 15 patients underwent other complicated simultaneous operations, including simultaneous hepatectomy, hysterectomy, adnexectomy, and other operations that significantly affect the operation time. Excluding these 15 cases, a total of 108 cases were included in this study. All patients were diagnosed with ultra-low rectal cancer before operation, and no invasion of external sphincter, levator ani and anorectal ring. This study was approved by the hospital ethics committee, and informed consent was obtained from patients and their families.

\section{Surgical techniques}

Referring to the literature [11], CSPO can be divided into abdominal operation and perineal operation.

Abdominal operation: first, the sigmoid colon was freed and the root of mesenteric artery was ligated. Then, according to the principle of TME, the rectum is freed to the level of hiatal ligament, the hiatal ligament is cut off, and the dissection is stopped at the entrance of sphincter space, which is one of the important differences between CSPO and Intersphincteric resection (ISR). During the operation, the autonomic nerve was protected and the intestinal canal was severed at the junction of rectum and sigmoid colon. 
Perineal operation: first, the anus was enlarged to 3-4 finger widths, and the rectum was prolapsed through the anus. Then, the conformal resection line was designed according to the location and size of the tumor. The key to the operation is to preserve the rectal wall, dentate line and internal sphincter on the opposite side of the tumor as much as possible on the premise of ensuring that the distal resection margin under direct vision is $\geq 1 \mathrm{~cm}$. In addition, avoid separating the sphincter space to prevent damage of the nerve tissue in it, and protect the function of the remaining sphincter as much as possible. In the case that the rectum can't be prolapsed through the anus, conformal resection of tumor can be performed by transanal first. Then the specimen was pulled out and the rectal stump was sutured intermittently. Routine intraoperative frozen section diagnosis was performed to ensure the safety of the distal resection margin and sufficient circumferential resection margin (CRM). After closing the rectal stump, a $25 \mathrm{~mm}$ tubular stapler (CDH25, Johnson \& Johnson, USA) was placed through the anus for anastomosis. The anastomosis position was selected on the side with more rectum, and the dentate line and internal sphincter on the opposite side of the tumor were retained as much as possible. If the rectal stump is too short, manual anastomosis can also be used (First, one 3-0 absorbable thread was used for upper, lower, left and right stitches, and then two 3-0 barbed threads were used for continuous suture, each thread was sutured half a circle).

All patients underwent laparoscopic surgery and temporarily ileumstomy, which were completed by the same surgeon and the same operation team.

\section{Statistical analysis}

The statistical analysis of this study is based on SPSS 23.0 statistical software, the moving average curve is drawn by Excel, and the CUSUM curve is drawn and fitted by SPSS 23.0 statistical software. The counting data were expressed by the rate (\%), and the normal distribution measurement data were expressed by means $\pm S D$, and those data were all accurate to the last decimal point. $\chi^{2}$ test or Fisher exact probability method was used to compare the counting data between groups; $t$ test was used to compare the measurement data between the two groups. $\mathrm{P}<0.05$ was considered to indicate a statistically significant difference between the data sets.

\section{Results}

\section{Baseline characteristics}

A total of 108 patients were included in this study. CSPO operation time $147 \pm 8 \mathrm{~min}$. The preoperative status of patients was evaluated by ASA-classification. The number of harvested lymph nodes was the number of lymph nodes detected by postoperative pathology. The nature and staging of the tumor were all postoperative staging. Therefore, some patients with pathological complete response (pCR) after nCRT were classified as non tumor / scar group, the degree of differentiation was classified as no mass / premalignant lesion group, and T stage was classified as TO group. Postoperative complications were defined as complications occurred within 30 days after operation, which were scored according to Clavien-Dindo classification. Postoperative infection was defined as fever with body temperature higher 
than $38{ }^{\circ} \mathrm{C}$ and hemogram rising within 30 days after operation, which could be relieved after antibiotic therapy(Table1).

\section{Learning curve}

Based on the moving average curve of CSPO operation time (Figure 1), it can be seen that with the accumulation of operation cases, the moving average is gradually decreasing, and the first lowest point appears at 57 cases. Although the curve fluctuated after that, it gradually stabilized. Combined with the lag effect of moving average, it can be predicted that there is a learning curve in CSPO, and the cut-off point value is before 57 cases.

The CUSUM scatter plot of CSPO operation time (Figure 2) was fitted with quadratic and cubic curves, and the equations were obtained: $Y=87.09+34.13 X-0.33 X^{2}, R^{2}=0.915 ; Y=-86.94+52.86 X-0.76 X^{2}+2.62-$ $3 X^{3}, R^{2}=0.957$. The goodness of fit of the cubic equation is higher, and the cut-off point value $X=45.4654$. According to the cut-off point value, the CSPO learning curve can be divided into learning period (1-45 cases) and learning completion period (46-108 cases).

\section{Comparison of baseline characteristics of patients in different periods}

There were no significant differences in preoperative clinical baseline data (gender, age, BMI, ASAclassification, previous abdominal surgical history, neoadjuvant therapy, CEA, CA19-9); surgical and postoperative pathological data (the number of harvested lymph nodes, distance of tumor from anal verge, distance of distal resection margin, maximum diameter of mass, tumor type, the degree of tumor differentiation, TNM staging); postoperative complications and survival data (postoperative complications, postoperative infection, classification of complications, and progression-free survival (PFS) rate in 3 years). However, the intraoperative blood loss in the learning period were significantly greater than those in the learning completion period, and the difference was statistically significant $(P<0.05)$. Besides, the flatus and defecation time, the liquid diet time and the postoperative hospital stay in the learning period were significantly longer than those in the learning completion period, and the difference was statistically significant $(P<0.05)(T a b l e 1)$.

\section{Univariate and multivariate analysis of influencing factors of CSPO operation time}

In order to explore the influencing factors of the operation time of CSPO, and to provide the basis for beginners to select the appropriate cases, the operation time of CSPO was further divided into three groups according to the 25 th percentile and 75th percentile values. Group $A$ and group $C$ were compared with group $B$ for univariate and multivariate analysis. The results of univariate analysis $(P<0.05)$ were included in multivariate analysis. The results of multivariate analysis $(P<0.05)$ were considered as independent risk factors affecting the operation time of CSPO (Table2-3). 
Multivariate analysis showed that distance of tumor from anal verge ( $\geq 4 \mathrm{~cm}$ vs. $<4 \mathrm{~cm}, P=0.039$ ) and T stage (T3 vs. T1-2, $P=0.022$ ) were independent risk factors for prolonged operation time of CSPO.

\section{Discussion}

Although CSPO is also divided into transabdominal and transanal surgery, it is different from ISR [20] or ultra-low Dixon. The first is to retain the internal anal sphincter and dentate line as much as possible. Then, the design of tumor resection line and conformal resection were carried out on the premise of the safety of tumor distal margin. In addition, the mechanical suture and manual suture of the excised conformal bowel are also different. These are not only the key reasons for the increased difficulty of CSPO, but also the key points need to be overcome in the process of learning and training. Therefore, the definition of CSPO learning curve is crucial for beginners to formulate training plan, select appropriate cases and set phased objectives. In addition, it also concerns the further promotion of CSPO.

Compared with previous studies on learning curve [21], this study explores CSPO learning curve through moving average, CUSUM, which are two more accurate statistical methods. It not only proved the existence of CSPO learning curve, but also defined the cut-off point of learning curve in 45 cases. According to this, the patients were divided into learning period and learning completion period. Subsequent statistical analysis proved that there was no significant difference between the two groups in curative resection (distance of distal margin, number of harvested lymph nodes), postoperative complications and prognosis. This reflects the safety of learning period, and the surgical effect and prognosis will not be affected by surgical proficiency. Therefore, beginners can actively try this operation. In addition, it also proved that the learning completion period not only achieved a significant reduction in the operation time and intraoperative bleeding, but also achieved a faster recovery of postoperative gut function and a shorter postoperative hospital stay. This is not solely related to the improvement of surgical proficiency, but also related to the enhancement of the cooperation ability of the overall operation team, which also reflects the concept of fast-track surgery [22].

This study also further investigated the influencing factors of CSPO operation time, in order to use our experience in CSPO to help beginners choose the appropriate cases and get through the learning curve faster.

Many previous studies have proved that obesity is an important risk factor for prolonged operation time [23]. Especially for ultra-low rectal cancer, obesity and narrow pelvis will increase the difficulty of free and anastomosis, thus increasing the operation time [24]. However, this study found that BMI was not an independent risk factor affecting the operation time of CSPO. On the one hand, this may be related to the bias between patient selection and enrollment in this study. That is, when doctors perform CSPO, the obesity degree is also a potential enrollment tendency. It can be seen that the body types of patients in this study are relatively moderate, and the BMl value is $23.0 \pm 3.3 \mathrm{~kg} / \mathrm{m}^{2}$. On the other hand, it is related to the fact that CSPO does not require cutting and anastomosis in the abdominal cavity. That is, the anastomosis process of coloanal canal is carried out through the anus, which breaks through the 
limitation of the patient's narrow pelvic space [25]. This reduces the impact of obesity on the operation time, which is also the advantage of CSPO.

At present, neoadjuvant therapy combined with TME and postoperative consolidation chemotherapy has become the standard treatment mode for advanced rectal cancer recommended by NCCN and ESMO guidelines. For ultra-low rectal cancer, nCRT can not only reduce the tumor size and the difficulty of operation, but also lower the tumor stage and achieve the criteria of anus preservation. However, many studies have shown that radiotherapy can lead to rectal wall fibrosis [26-27]. This fibrosis will disturb the normal anatomical layer of perianal tissue, reduce the tactile feedback, increase intraoperative bleeding, prolong the operation time, reduce the postoperative anal function [28-29]. However, multivariate analysis in this study showed that nCRT was not an independent risk factor affecting the operation time. On the one hand, this is because the sphincter space is dissected through the anus under direct vision, which can facilitate hemostasis and clarity of anatomical layer, which is also the advantage of CSPO. On the other hand, many studies have shown that nCRT will cause microscopic morphological changes of internal anal sphincter [30-31], but have little effect on its macroscopical morphology. Therefore, as long as we pay attention to the anatomical layer, the difficulty of operation will not increase too much.

In terms of the distance of tumor from anal verge, this study showed that the distance of tumor from anal verge $\geq 4 \mathrm{~cm}$ was an independent risk factor affecting the operation time of CSPO. This is different from the previous cognition that the closer the tumor is to the anal margin, that is, the lower the tumor location, the greater the difficulty of operation. There are several reasons. Firstly, the higher the tumor location, the more difficult it is to expose the limited space through anus under direct vision, which is easy to bleed and confuse the anatomical layer, increasing the difficulty. In addition, the patients included in this study were all with ultra-low rectal cancer whose lower edge of the tumor less than $2 \mathrm{~cm}$ from the dentate line. The distance of tumor from anal verge $\geq 4 \mathrm{~cm}$ means that the patient's anal canal is longer and the tumor is deeper under direct vision, which is also related to the patient's obesity. These will increase the difficulty of surgery. This is a significant difference between CSPO and other rectal cancer surgery, and also shows the advantage that CSPO can achieve anal preservation in a lower position.

In terms of tumor staging, all patients included in this study met the criteria of type II and III of rullier classification [32]. It is required that the external anal sphincter of the patients is not invaded, so all the enrolled patients are T1-3 stage patients. Consistent with previous studies, multivariate analysis showed that T3 stage was an independent risk factor for CSPO operation time. On the one hand, the late tumor stage is often accompanied by nCRT for a long time, which has a certain impact on the operation. On the other hand, many previous studies have shown that the T stage of the tumor, that is, the depth of tumor invasion, is significantly and independently related to the local recurrence of the tumor [33]. In addition, late $T$ stage is related to the distal invasion, lateral invasion and lymph node metastasis. Therefore, for patients with late $T$ stage, surgeons often need to clean lymph nodes and perform local operations more carefully, which will prolong operation time. 
There are still some limitations in this study. First, this is a single center non random retrospective study, so there are some biases and deficiencies in the design itself. Second, this study mainly focuses on one surgeon's experience, and the operative quantity is not particularly large. Considering the heterogeneity between surgeons, other surgeons may have different results in CSPO. In addition, the surgeon in this study has accumulated experience in more than 100 cases of laparoscopic surgery, so the CSPO learning curve may be longer for novice surgeons. Third, this study takes the operation time as the main index to measure the operator's experience accumulation, but in fact, there are many other factors that may affect the operation time. In addition to the operation time, other indicators, such as the amount of bleeding, can also be used to reflect the operator's experience. Fourth, the prolongation of liquid diet time and hospital stay in the learning period may be partly due to the selection bias of the researchers. Because more conservative strategies are often adopted in the early stage of a new operation to ensure its safety. Fifth, the cut-off value of learning curve and the results of univariate and multivariate analysis need to be further proved in clinical practice. For these reasons, a prospective, multi-center and multi-index randomized controlled study is needed to further confirm the conclusions of this study and explore the methods to shorten the learning curve.

\section{Conclusions}

This study not only proved the existence of CSPO learning curve by moving average and CUSUM methods, but also further clarified that surgeons with laparoscopic surgery experience need 45 cases of CSPO to complete the learning curve. In addition, further univariate and multivariate analysis showed that distance of tumor from anal verge ( $\geq 4 \mathrm{~cm}$ vs. $<4 \mathrm{~cm}, P=0.039$ ) and T stage (T3 vs. T1-2, $P=0.022$ ) were independent risk factors for prolonging the operation time of CSPO. The experience of CSPO in our center is that the key steps are transabdominal laparoscopic operation and transanal operation. Therefore, it is suggested that beginners should strengthen their understanding of perianal anatomy, and have some experience in laparoscopic surgery, perianal disease surgery and manual coloanal anastomosis technology. Initially, it is recommended to select patients with benign or early malignant tumors (<T3) whose tumor is less than $4 \mathrm{~cm}$ from the anal verge, and then further select more complex cases after about 45 cases of CSPO. In this way, beginners can reduce the operation time, accumulate experience, build self-confidence and shorten the learning curve on the premise of safety.

\section{Abbreviations}

CSPO

Conformal sphincter preservation operation CUSUM

Cumulative sum control chart

nCRT

neoadjuvant radiotherapy and chemotherapy

TME 
Total mesorectal excision

NOTES

Natural orifice translumenal endoscopic surgery

ISR

Intersphincteric resection

CRM

Circumferential resection margin

pCR

Pathological complete response

PFS

Progression-free survival

NCCN

National Comprehensive Cancer Network

ESMO

European Society for Medical Oncology.

\section{Declarations}

\section{Acknowledgements}

The authors thank Jian-rong Zhang of Anhui Agricultural University for editing.

\section{Authors' contributions}

HD, LW, GS and GY contributed to the study design. HD , LW, GS and GY wrote the manuscript. GY, XG, HG, JS, XZ, WZ revised the manuscript. HD, GS, and KZ collected the data. HD, LW, and GS analyzed the data. All authors read and approved the final manuscript.

\section{Funding}

This study was supported by the "234 Subjects Peak Climbing Plan" of the First Affiliated Hospital of Naval Medical University (2019YXK032).

\section{Availability of data and materials}

The datasets used and analyzed during the current study are available from the corresponding author on reasonable request.

\section{Ethics approval and consent to participate}

Ethical permission for this study was obtained from the Institutional Review Board, and the written informed consents were acquired from all patients. 
Not applicable.

\section{Competing interests}

The authors declare that they have no competing interests.

\section{References}

1. Nissan A, Guillem JG, Paty PB, Wong WD, Minsky B, Saltz L, et al. Abdominoperineal resection for rectal cancer at a specialty center. Dis Colon Rectum. 2001;44(1):27-35.

2. Krishnamurty DM, Blatnik J, Mutch M. Stoma Complications. Clin Colon Rectal Surg. 2017;30(03):193-200.

3. Gooszen AW, Geelkerken RH, Hermans J, Lagaay MB, Gooszen HG. Quality of life with a temporary stoma: Ileostomy vs. colostomy. Dis Colon Rectum. 2000;43(5):650-655.

4. Yau T, Watkins D, Cunningham D, Barbachano Y, Chau I, Chong G. Longitudinal assessment of quality of life in rectal cancer patients with or without stomas following primary resection. Dis Colon Rectum. 2009;52(4):669-677.

5. Zolciak A, Bujko K, Kepka L, Oledzki J, Rutkowski A, Nowacki MP. Abdominoperineal resection or anterior resection for rectal cancer: Patient preferences before and after treatment. Color Dis. 2006;8(7):575-80.

6. Fokas E, Allgäuer M, Polat B, Klautke G, Grabenbauer GG, Fietkau R, et al. Randomized phase II trial of chemoradiotherapy plus induction or consolidation chemotherapy as total neoadjuvant therapy for locally advanced rectal cancer: CAO/ArO/AIO-12. J Clin Oncol. 2019;37(34):3212-22.

7. Dossa F, Acuna SA, Rickles AS, Berho M, Wexner SD, Quereshy FA, et al. Association between adjuvant chemotherapy and overall survival in patients with rectal cancer and pathological complete response after neoadjuvant chemotherapy and resection. JAMA Oncol. 2018;4(7):930-7.

8. Keranmu A, Liu HN, Wu YC, Liu TT, Li C, Guo TA, et al. A negative-doughnut distal resection margin less than $5 \mathrm{~mm}$ does not affect prognosis in rectal cancer. J Surg Oncol. 2018;118(3):536-43.

9. Lim SB, Heo SC, Lee MR, Kang SB, Park YJ, Park KJ, et al. Changes in outcome with sphincter preserving surgery for rectal cancer in Korea, 1991-2000. Eur J Surg Oncol. 2005;31(3):242-9.

10. Yamada K, Saiki Y, Takano S, Iwamoto K, Tanaka M, Fukunaga M, et al. Long-term results of intersphincteric resection for low rectal cancer in Japan. Surg Today. 2019;49(4):275-285.

11. Sun G, Lou Z, Zhang H, Yu GY, Zheng K, Gao XH, et al. Retrospective study of the functional and oncological outcomes of conformal sphincter preservation operation in the treatment of very low rectal cancer. Tech Coloproctol. 2020;24(10):1025-34.

12. Lou Z, Zhang W. Transanal conformal resection for super low rectal cancer. Zhonghua Wei Chang Wai Ke Za Zhi. 2018;21(3):246-9.

13. Zhu X, Lou Z, Bai C, Gong H, Ma J, Meng R, et al. Preliminary investigation of intramural lateral spread distance in pull-through conformal resection of low rectal cancer. Zhonghua Wei Chang Wai 
Ke Za Zhi. 2016;19(9):1025-9.

14. Palanivelu C, Sendhilkumar K, Jani K, Rajan PS, Maheshkumar GS, Shetty R, et al. Laparoscopic anterior resection and total mesorectal excision for rectal cancer: A prospective nonrandomized study. Int J Colorectal Dis. 2007;22(4):367-72.

15. Kwok SPY, Lau WY, Declan Carey P, Kelly SB, Leung KL, Li AKC. Prospective evaluation of laparoscopic-assisted large-bowel excision for cancer. Ann Surg. 1996;223(2):170-6.

16. Denost Q, Adam JP, Pontallier A, Celerier B, Laurent C, Rullier E. Laparoscopic total mesorectal excision with coloanal anastomosis for rectal cancer. Ann Surg. 2015:138-43.

17. Yeh YS, Chen MJ, Tsai HL, Huang MY, Chen CW, Huang YH, et al. Transanal inside-out rectal resection for ultra-low rectal cancer. J Investig Surg. 2012;25(6):375-80.

18. Veereman G, Vlayen J, Robays J, Fairon N, Stordeur S, Rolfo C, et al. Systematic review and metaanalysis of local resection or transanal endoscopic microsurgery versus radical resection in stage $\mathrm{i}$ rectal cancer: A real standard? Crit Rev Oncol Hematol. 2017; 114: 43-52.

19. Marks JH, Lopez-Acevedo N, Krishnan B, Johnson MN, Montenegro GA, Marks GJ. True NOTES TME resection with splenic flexure release, high ligation of IMA, and side-to-end hand-sewn coloanal anastomosis. Surg Endosc. 2016;30(10):4626-31.

20. Sun G, Zang Y, Ding H, Chen Y, Gong H, Lou Z, et al. Clinical efficacy of conformal sphincter preservation operation versus intersphincteric resection in the treatment of low rectal cancer. Chinese J Dig Surg. 2021;20(3):292-300.

21. Lu J, Huang CM, Zheng CH, Li P, Xie JW, Wang J Bin, et al. Learning curve of laparoscopy spleenpreserving splenic hilar lymph node dissection for advanced upper gastric cancer. Hepatogastroenterology. 2013;60(121):296-300.

22. Muller S, Zalunardo MP, Hubner M, Clavien PA, Demartines N. A fast-track program reduces complications and length of hospital stay after open colonic surgery. Gastroenterology. 2009;136(3):842-7.

23. Tjeertes EEKM, Hoeks SSE, Beks SSBJC, Valentijn TTM, Hoofwijk AAGM, Stolker RJRJ. Obesity - a risk factor for postoperative complications in general surgery? BMC Anesthesiol. 2015;15(1):1-7.

24. Ding H, Li J, Chen Y, Yang Z, Peng Z, Liao X. Anal function and quality of life analysis after laparoscopic modified Parks for ultra-low rectal cancer patients. World J Surg Oncol. 2020;18(1).

25. Atasoy G, Arslan NC, Elibol FD, Sagol O, Obuz F, Sokmen S. Magnetic resonance-based pelvimetry and tumor volumetry can predict surgical difficulty and oncologic outcome in locally advanced midlow rectal cancer. Surg Today. 2018;48(12):1040-51.

26. Chen FC, Mackay JR, Woods RJ, Collopy BT, Fink RJ, Guiney MJ. EARLY EXPERIENCE WITH POSTOPERATIVE ADJUVANT CHEMORADIATION FOR RECTAL CARCINOMA: FOCUS ON MORBIDITY. Aust N Z J Surg. 1995;65(10):732-6.

27. Kollmorgen CF, Meagher AP, Wolff BG, Pemberton JH, Martenson JA, Ilstrup DM. The long-term effect of adjuvant postoperative chemoradiotherapy for rectal carcinoma on bowel function. Ann Surg. 1994;220(5):676-82. 
28. Desnoo L, Faithfull S. A qualitative study of anterior resection syndrome: The experiences of cancer survivors who have undergone resection surgery. Eur J Cancer Care (Engl). 2006;15(3):244-51.

29. Putta S, Andreyev HJN. Faecal incontinence: A late side-effect of pelvic radiotherapy. Clin Oncol. 2005;17(6):469-77.

30. Da Silva GM, Berho M, Wexner SD, Efron J, Weiss EG, Nogueras JJ, et al. Histologic Analysis of the Irradiated Anal Sphincter. Dis Colon Rectum. 2003;46(11):1492-7.

31. Nishizawa Y, Fujii S, Saito N, Ito M, Ochiai A, Sugito M, et al. The association between anal function and neural degeneration after preoperative chemoradiotherapy followed by intersphincteric resection. Dis Colon Rectum. 2011;54(11):1423-9.

32. Rullier E, Denost Q, Vendrely V, Rullier A, Laurent C. Low rectal cancer: Classification and standardization of surgery. Dis Colon Rectum. 2013;56(5):560-7.

33. Masaki T, Matsuoka H, Kishiki T, Kojima K, Tonari A, Aso N, et al. Site-specific risk factors for local recurrence after rectal cancer surgery. Surg Oncol. 2021;37.

\section{Tables}

Due to technical limitations, Tables are only available as a download in the Supplemental Files section.

\section{Figures}

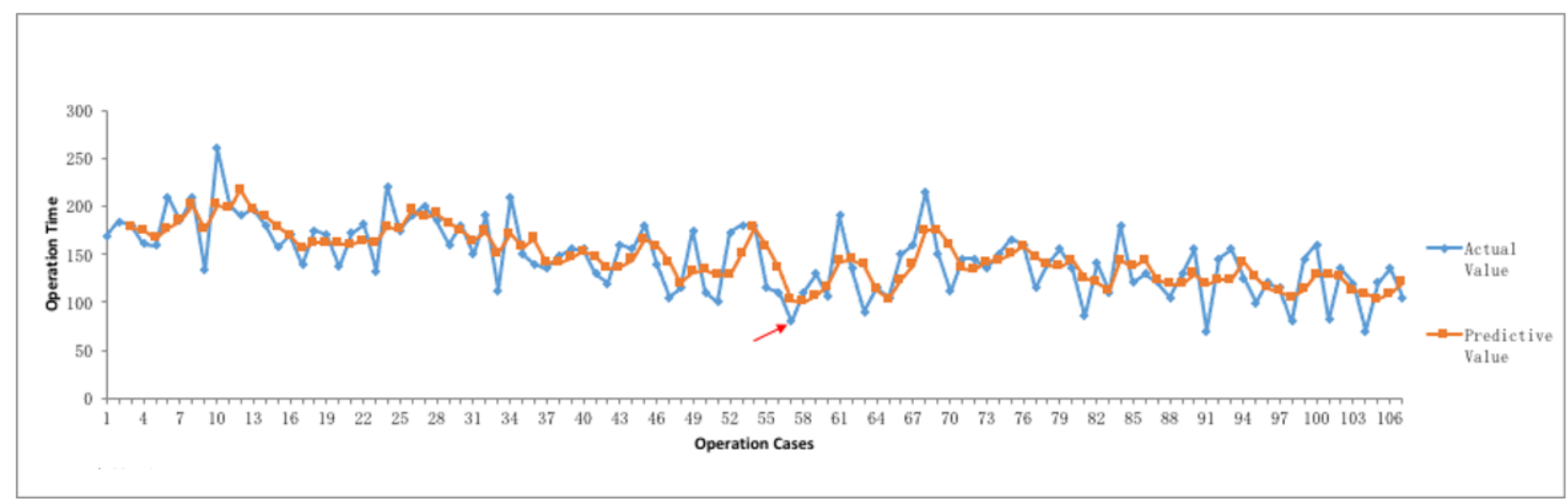

\section{Figure 1}

Moving average curve of CSPO operation time $\rightarrow$ The first lowest point of the moving average curve 


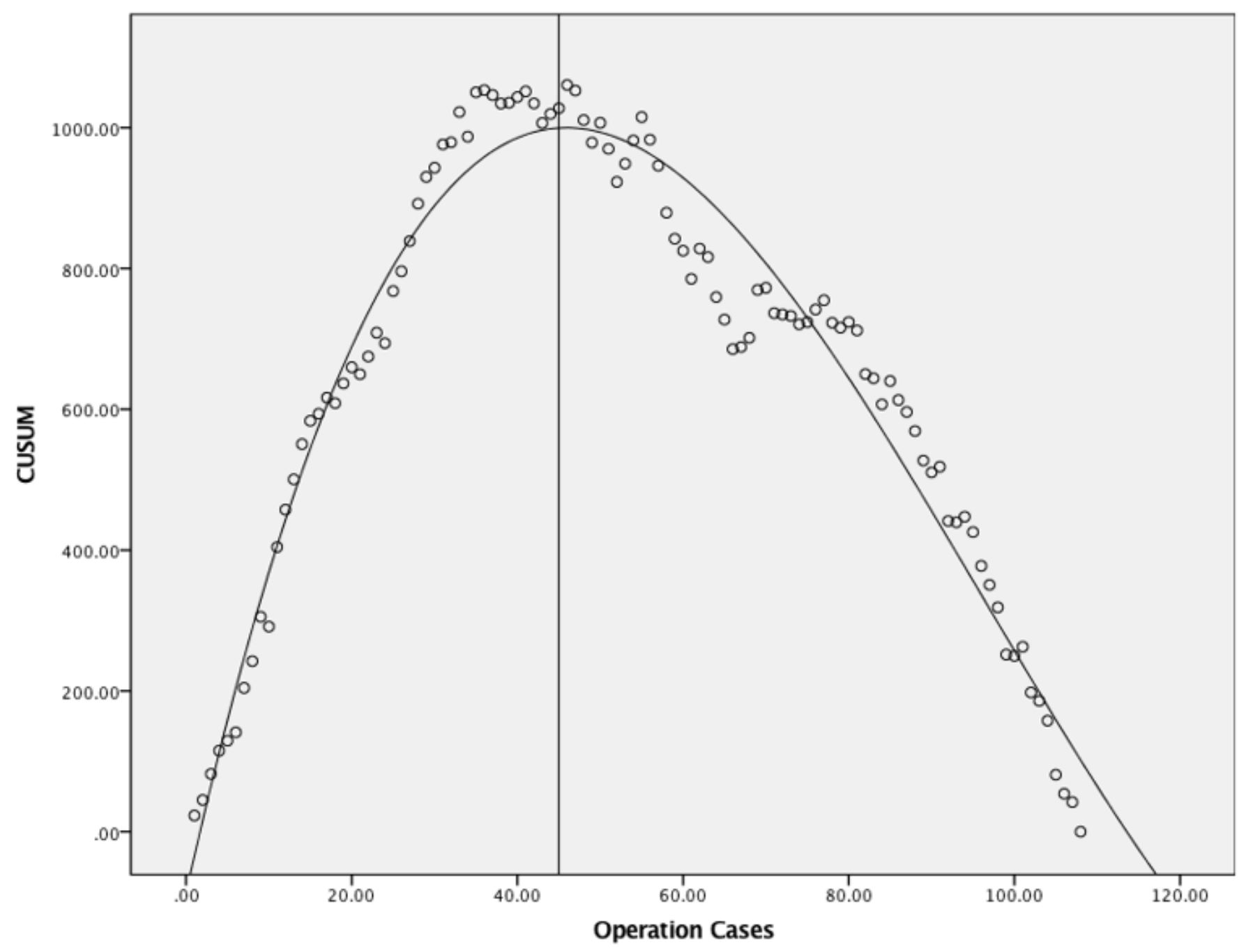

Figure 2

CUSUM curve of CSPO operation time $₫$ Vertical line through CUSUM curve cut-off point

\section{Supplementary Files}

This is a list of supplementary files associated with this preprint. Click to download.

- Learningcurvetables.xls 